Check for updates

Cite this: RSC Adv., 2017, 7, 33580

Received 23rd May 2017

Accepted 27th June 2017

DOI: 10.1039/c7ra05774a

rsc.li/rsc-advances

\section{Eutypellazines A-M, thiodiketopiperazine-type alkaloids from deep sea derived fungus Eutypella sp. MCCC 3A00281†}

\begin{abstract}
Siwen Niu, ${ }^{\text {ab }}$ Dong Liu, ${ }^{a}$ Zongze Shao, ${ }^{\text {tb }}$ Peter Proksch ${ }^{c}$ and Wenhan Lin (DD *a
Bioassay in association with the NMR/MS spectroscopic data guided fractionation of the solid fermentation of a deep sea derived fungus Eutypella sp. MCCC 3A00281, resulted in the isolation of 13 new thiodiketopiperazine-type alkaloids, namely eutypellazines A-M (1-13). Their structures were elucidated on the basis of extensive spectroscopic data analysis, including the ECD data, modified Mosher's method, and the $\mathrm{Cu}-\mathrm{K} \alpha$ X-ray single-crystal diffraction experiments for the determination of the absolute configurations. An anti-HIV bioassay indicated that compounds 1-12 exhibited inhibitory effects against pNL4.3.Env-.Luc co-transfected 293T cells (HIV-1 model cells) with low cytotoxicity, of which eutypellazine $E$ exerted the highest activity. A preliminary structure-activity relationship was discussed. In addition, eutypellazine J (10) and epicoccin A showed reactivating effects toward latent HIV-1 in J-Lat A2 cells.
\end{abstract}

\section{Introduction}

The natural thiodiketopiperazine alkaloids (TDKPs) are a class of unique fungal secondary metabolites, which are characterized by the presence of a diketopiperazine core featuring thiomethyl groups and/or transannular sulfide bridges. ${ }^{1-6}$ The TDKP derivatives are biogenetically derived from at least one aromatic amino acid (phenylalanine, tryptophan or tyrosine), while the unusual sulfur bridges and thiomethyl units are generated by the mediation of biosynthetic genes such as glutathione S-transferase. ${ }^{7,8}$ TDKP derivatives are widely distributed in diverse fungal genera, whereas the fungal strains, belonging to the genera of Penicillium, ${ }^{6}$ Exserohilum, ${ }^{9}$ and $P$ homa ${ }^{10}{ }^{10}$ from marine inhabitation are the rich sources to derive TDKP analogues. So far, an array of TDKPs with diverse scaffolds due to the formation of different amino acids or distinct sulfur bridges has been found from fungal origin, and the structural variety directly affected their bioactivity. The biological activities are mainly focused on cytotoxic,, ${ }^{, 11}$ antibacterial, ${ }^{12,13}$ antiangiogenic, ${ }^{14}$ anti-inflammatory, ${ }^{15}$ and antituberculosis. ${ }^{16}$ In continuation of our research program aiming the discovery of bioactive metabolites from deep-sea derived microorganisms, the EtOAc extract of the fermentation of a deepsea derived fungus Eutypella sp. MCCC 3A00281 exhibited

${ }^{a}$ State Key Laboratory of Natural and Biomimetic Drugs, Peking University, Beijing, 100191, P. R. China. E-mail: whlin@bjmu.edu.cn; Fax: +86-10-82806188

${ }^{b}$ Key Laboratory of Marine Biogenetic Resources, Third Institute of Oceanography, SOA, Xiamen, 361005, P. R. China

'Institute of Pharmaceutical Biology and Biotechnology, Heinrich-Heine University, 40225 Duesseldorf, Germany

$\dagger$ Electronic supplementary information (ESI) available. CCDC 1416589-1416593. For ESI and crystallographic data in CIF or other electronic format see DOI: 10.1039/c7ra05774a inhibitory effect against HIV-1 virus (Table 1). Chromatographic fractionation of the active extract in association with the bioassay and NMR/ESIMS experiments revealed that the active fractions F7 and F8 featured a profile of thiodiketopiperazine-type derivatives. Further chromatographic separation of both F7 and F8 fractions resulted in the isolation of 15 TDKP derivatives, including 13 new analogues namely eutypellazines A-M (1-13) (Fig. 1). In this paper, we intend to report the structure elucidation of new compounds and their anti-HIV-1 activities.

\section{Experimental section}

\subsection{General procedure}

Melting points were measured on X-5 micromelting-point apparatus. IR spectra were determined by a Thermo Nicolet

Table 1 Inhibitory effects of the fractions toward HIV $-1^{a}$

\begin{tabular}{lll}
\hline Fractions & $c\left(\mu \mathrm{g} \mathrm{mL}^{-1}\right)$ & Inhibitory rates $(\%)$ \\
\hline EtOAc extract & 10 & 46.3 \\
F3 & 10 & 0 \\
F4 & 10 & 9.7 \\
F5 & 10 & 29.4 \\
F6 & 10 & 5.1 \\
F7 & 10 & 99.6 \\
F8 & 10 & 68.6 \\
F9 & 10 & 46.0 \\
F10 & 10 & 18.0 \\
EFV & $0.1(\mu \mathrm{M})$ & 96.22
\end{tabular}

${ }^{a}$ EFV (Efavirenz): positive control, bioassay was performed in pNL4.3.Env-.Luc co-transfected 293T cells. 

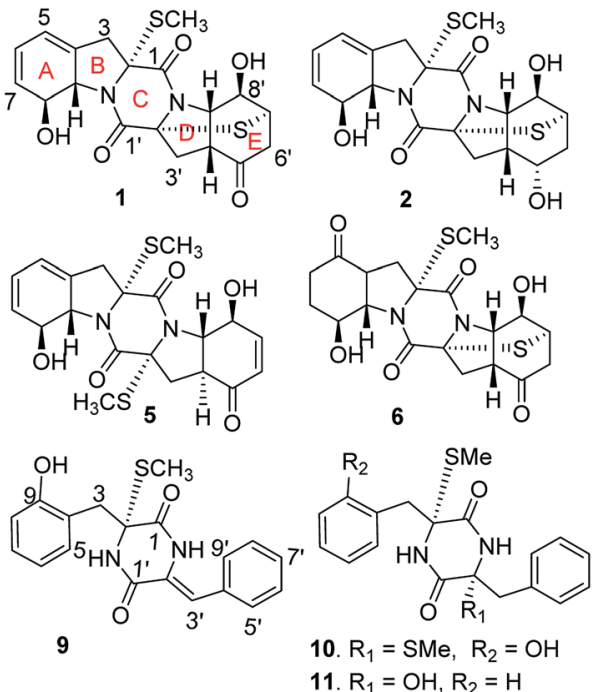

11. $\mathrm{R}_{1}=\mathrm{OH}, \mathrm{R}_{2}=\mathrm{H}$
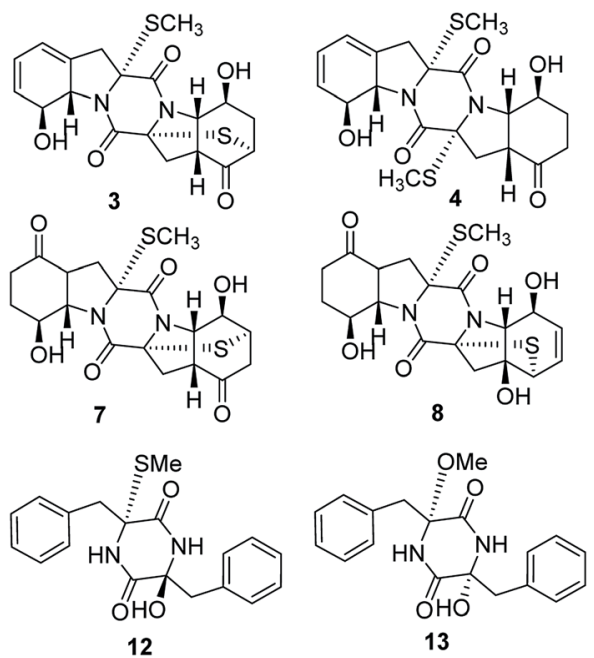

13

Fig. 1 Structures of new compounds.

Nexus 470 FT-IR spectrometer. UV spectra were measured on a Cary 300 spectrometer. Optical rotations were obtained from a Rudolph IV Autopol automatic polarimeter at $25{ }^{\circ} \mathrm{C}$. CD spectra were measured on a JASCO J-810 spectropolarimeter. NMR spectra were recorded on Bruker Advance $400 \mathrm{MHz}$ spectrometers. Chemical shifts are expressed in $\delta(\mathrm{ppm})$ referenced to the solvent peaks at $\delta_{\mathrm{H}} 2.50 / \delta_{\mathrm{C}} 39.5$ for DMSO- $d_{6}$ and $\delta_{\mathrm{H}} 7.26 /$ $\delta_{\mathrm{C}} 77.2$ for $\mathrm{CDCl}_{3}$, and coupling constants $(J)$ are in Hz. HRESIMS spectra were obtained from Xevo G2 Q-TOF mass spectrometer (Waters). X-ray data were obtained by a Bruker D8 Advance single crystal X-ray diffractometer using graphite monochromated $\mathrm{Cu}-\mathrm{K} \alpha$ radiation. Column chromatography (CC) was performed by silica gel (100-200 and 200-300 mesh, Qingdao Marine Chemistry Co. Ltd. China), ODS gel (50 $\mu \mathrm{m}$, YMC, Japan) and Sephadex LH-20 (18-110 $\mu \mathrm{m}$, Amersham Pharmacia Biotech AB, Uppsala, Sweden). The precoated silica gel plates (Merck, Kieselgel $60 \mathrm{~F} 254,0.25 \mathrm{~mm}$ ) were used for TLC analysis. HPLC chromatography was performed on a Waters e2695 separation Module coupled with a Waters 2998 photodiode array detector, and a semi-preparative reversedphased column (YMC-packed C18, $5 \mu \mathrm{m}, 250 \mathrm{~mm} \times 10 \mathrm{~mm}$ ) was used for purification. 293T cells (ATCC® CRL-3216 ${ }^{\mathrm{TM}}$ ) from embryonic kidney of human were provided from American Type Culture Collection (ATCC). J-lat-A2 cells were provided by the Verdin laboratory (Buck Institute, CA), and were generated by transduction of Jurkat T cells (NIH AIDS Reagent Program) with an HIV vector expressing Tat-Flag and GFP under the control of the viral $5^{\prime}$-LTR and IRES positioned in between Tat and GFP (LTR-Tat-Flag-IRES-GFP).

\subsection{Fungal material and fermentation}

The fungus Eutypella sp. MCCC 3A00281 was isolated from the deep sea sediment, collected with TV-multicore from South Atlantic Ocean (GPS $27.90 \mathrm{~W}, 6.43 \mathrm{~S}$ ) at the depth of 5610 meters during the Comra 22nd oceanic cruise in June 2011. On the basis of the rRNA analysis, the genus of Eutypella was determined (GeneBank accession number KT366012). The strain was deposited in the Marine Culture Collection of China (MCCC), Third Institute of Oceanography, State Oceanic Administration (SOA), Xiamen, China.

The fungal strain MCCC 3A00281 was cultured on the slants of PDA medium at $25^{\circ} \mathrm{C}$ for 6 days. The fresh mycelia were cut and inoculated to $45 \times 500 \mathrm{~mL}$ Erlenmeyer flasks, each flask contains $100 \mathrm{~g}$ rice and $140 \mathrm{~mL}$ distilled water. After autoclaving at 15 psi for $30 \mathrm{~min}$, these flasks were inoculated and incubated at room temperature for 25 days. The fermented fungal material was extracted with EtOAc for three times, and then concentrated under vacuo to afford crude extract.

\subsection{Isolation and purification}

The EtOAc extract was tested by the pNL4.3.Env-.Luc cotransfected 293T cells (HIV-1 model cells) showing 46\% inhibitory rate in a dose of $10 \mu \mathrm{g} \mathrm{mL} \mathrm{m}^{-1}$. The active extract (13 g) was subjected to a silica gel vacuum liquid chromatography (VLC), eluting with a gradient of petroleum ether (PE) and acetone $(1: 0$ to $0: 1$ ) to yield 10 fractions (F1-F10). Fractions F1 to F4 (sum amount $4.3 \mathrm{~g}$ ) were detected by the ${ }^{1} \mathrm{H}$ NMR spectra to show the resonances mainly containing fatty acids. Fractions F5 and F6 (sum amount $2.0 \mathrm{~g}$ ) featured terpenoid resonances in the ${ }^{1} \mathrm{H}$ NMR spectra. The polar fractions F9 and F10 (sum amount $5.4 \mathrm{~g}$ ) displayed the resonances for polyphenols and saccharides in the ${ }^{1} \mathrm{H}$ NMR spectra. In addition, the ${ }^{1} \mathrm{H}$ NMR and ESIMS spectra of F7 and F8 (sum amount $1.0 \mathrm{~g}$ ) displayed the metabolite profile of thiodiketopiperazine alkaloids. Bioassay of the fractions F3 to F10 using pNL4.3.Env-.Luc co-transfected 293T cells (HIV-1 model cells) revealed that F7 and F8 possess potential inhibition against HIV-1 virus, of which F7 showed the most active (Table 1). Fraction F7 (0.4 g) was subjected to ODS column chromatography (CC) eluting with a gradient with increasing $\mathrm{MeOH}$ in $\mathrm{H}_{2} \mathrm{O}(30 \%$ to $100 \%)$ to afford 11 subfractions (SF71SF711). The HPLC fingerprints of SF71-SF76 were overlapped with that of inactive fraction F6. SF77 (112 mg) was separated by 
the semi-preparative HPLC with a mobile phase of $\mathrm{MeCN}-\mathrm{H}_{2} \mathrm{O}$ (3 : 2) to obtain 5 (13.4 mg), 10 (2.8 mg), 4 (46.6 mg), and 9 (2.3 $\mathrm{mg})$. SF78 (157 mg) was separated by the semi-preparative HPLC with a mobile phase of $\mathrm{MeCN}-\mathrm{H}_{2} \mathrm{O}(7: 13)$ to obtain $13(15.8 \mathrm{mg})$ and 12 (4.5 mg). F8 (0.5 g) was fractionated upon an ODS column chromatography using a gradient elution of $\mathrm{MeOH}$ in $\mathrm{H}_{2} \mathrm{O}(20$ $100 \%$ ) to afford 15 subfractions (SF81-SF815). SF86 (101 mg) was separated by the semi-preparative HPLC $\left(\mathrm{MeCN}-\mathrm{H}_{2} \mathrm{O}, 1: 3\right)$ to afford epicoccin A (42.3 mg), 6 (28.8 mg), and 7 (14.2 mg), while 8 (2.5 mg) from SF88 (MeCN- $\left.\mathrm{H}_{2} \mathrm{O}=7: 18\right), 2$ (3.5 mg) from SF89 $\left(\mathrm{MeOH} / \mathrm{H}_{2} \mathrm{O}=43: 57\right)$, and $1(47.5 \mathrm{mg})$ from $\mathrm{SF} 811\left(\mathrm{MeCN} / \mathrm{H}_{2} \mathrm{O}\right.$ $=27: 73)$ were separated by the semi-preparative HPLC chromatography. SF812 (135 mg) was subjected to silica gel CC eluted with $\mathrm{CH}_{2} \mathrm{Cl}_{2}-\mathrm{MeOH}(50: 1)$ to yield $3(12.7 \mathrm{mg})$, epicoccin I (10.6 $\mathrm{mg})$, and 11 (2.6 mg).

Eutypellazine A (1). Monoclinic crystal; mp 267-269 ${ }^{\circ} \mathrm{C}$; $[\alpha]_{\mathrm{D}}^{25}+160(c 0.2, \mathrm{MeOH}) ; \mathrm{UV}(\mathrm{MeOH}) \lambda_{\max }(\log \varepsilon) 202(4.11)$ and 264 (3.55) nm; CD (MeOH) $\lambda_{\max }(\Delta \varepsilon) 267$ (+5.45), 246 (+7.10), 205 $(+20.13) \mathrm{nm}$; IR (KBr) $\nu_{\max } 3370,2922,1713,1647,1418,1324$, 1230, $1190 \mathrm{~cm}^{-1} ;{ }^{1} \mathrm{H}$ and ${ }^{13} \mathrm{C}$ NMR data, see Tables 2 and 4; HRESIMS $m / z 421.0887[\mathrm{M}+\mathrm{H}]^{+}$(calcd for $\mathrm{C}_{19} \mathrm{H}_{21} \mathrm{~N}_{2} \mathrm{O}_{5} \mathrm{~S}_{2}$, 421.0892).

Eutypellazine B (2). White powder; $[\alpha]_{\mathrm{D}}^{25}+140(c 0.1, \mathrm{MeOH})$; $\mathrm{UV}(\mathrm{MeOH}) \lambda_{\max }(\log \varepsilon) 204$ (4.01), 265 (3.06) nm; CD (MeOH) $\lambda_{\max }(\Delta \varepsilon) 264$ (+6.78), 245 (+5.45), 220 (+9.58), 201 (+11.38) nm; IR (KBr) $\nu_{\max } 3392,2920,1697,1649,1416,1232 \mathrm{~cm}^{-1} ;{ }^{1} \mathrm{H}$ and ${ }^{13} \mathrm{C}$ NMR data, see Tables 2 and 4; HRESIMS $m / z$ 423.1043 [M + $\mathrm{H}]^{+}$(calcd for $\mathrm{C}_{19} \mathrm{H}_{23} \mathrm{~N}_{2} \mathrm{O}_{5} \mathrm{~S}_{2}, 423.1048$ ).

Eutypellazine C (3). White powder; $[\alpha]_{\mathrm{D}}^{25}+277(c$ 0.2, $\mathrm{MeOH})$; $\mathrm{UV}(\mathrm{MeOH}) \lambda_{\max }(\log \varepsilon) 204$ (4.18), 266 (3.59) nm; CD (MeOH) $\lambda_{\max }(\Delta \varepsilon) 307$ (+8.45), $248(-3.19), 213(+16.65) \mathrm{nm}$; IR $(\mathrm{KBr}) \nu_{\max }$ 3366, 2921, 1706, 1648, 1418, $1230 \mathrm{~cm}^{-1} ;{ }^{1} \mathrm{H}$ and ${ }^{13} \mathrm{C}$ NMR data,
Tables 2 and 4; HRESIMS $m / z 421.0888[\mathrm{M}+\mathrm{H}]^{+}$(calcd for $\left.\mathrm{C}_{19} \mathrm{H}_{21} \mathrm{~N}_{2} \mathrm{O}_{5} \mathrm{~S}_{2}, 421.0892\right)$.

Eutypellazine D (4). White powder; $[\alpha]_{\mathrm{D}}^{25}-101(c 0.5, \mathrm{MeOH})$; UV (MeOH) $\lambda_{\max }(\log \varepsilon) 205$ (4.10), 261 (3.59) nm; CD (MeOH) $\lambda_{\max }(\Delta \varepsilon) 284(+1.60), 262(-3.72), 237(+4.33), 212(-8.36) \mathrm{nm}$; IR (KBr) $\nu_{\max } 3379,2923,1706,1637,1414,1328,1237,1201$ $\mathrm{cm}^{-1} ;{ }^{1} \mathrm{H}$ and ${ }^{13} \mathrm{C}$ NMR data, see Tables 2 and 4 ; HRESIMS $\mathrm{m} / \mathrm{z}$ 437.1201 $[\mathrm{M}+\mathrm{H}]^{+}$(calcd for $\mathrm{C}_{20} \mathrm{H}_{25} \mathrm{~N}_{2} \mathrm{O}_{5} \mathrm{~S}_{2}, 437.1205$ ).

Eutypellazine E (5). Orthorhombic crystal; mp 242-244 ${ }^{\circ} \mathrm{C}$; $[\alpha]_{\mathrm{D}}^{25}-154(c 0.5, \mathrm{MeOH}) ; \mathrm{UV}(\mathrm{MeOH}) \lambda_{\max }(\log \varepsilon) 207$ (4.16), 264 (3.55) nm; CD $(\mathrm{MeOH}) \lambda_{\max }(\Delta \varepsilon) 289(+1.59), 256(-2.48), 248$ (-2.27), 215 (-31.04) nm; IR (KBr) $\nu_{\max } 3351,2922,1692,1639$, 1412, 1374, 1262, 1185, $1125 \mathrm{~cm}^{-1} ;{ }^{1} \mathrm{H}$ and ${ }^{13} \mathrm{C}$ NMR data, see Tables 2 and 4; HRESIMS $m / z$ 435.1049 $[\mathrm{M}+\mathrm{H}]^{+}$(calcd for $\mathrm{C}_{20} \mathrm{H}_{23} \mathrm{~N}_{2} \mathrm{O}_{5} \mathrm{~S}_{2}$, 435.1048).

Eutypellazine F (6). Orthorhombic crystal; mp $208-210{ }^{\circ} \mathrm{C}$; $[\alpha]_{\mathrm{D}}^{25}+84(c 1.0, \mathrm{MeOH}) ; \mathrm{UV}(\mathrm{MeOH}) \lambda_{\max }(\log \varepsilon) 207$ (4.33) nm; IR (KBr) $\nu_{\max } 3396,2922,1709,1655,1419,1362,1324,1225 \mathrm{~cm}^{-1}$; ${ }^{1} \mathrm{H}$ and ${ }^{13} \mathrm{C}$ NMR data, see Tables 3 and 4 ; HRESIMS $\mathrm{m} / \mathrm{z}$ $439.0995[\mathrm{M}+\mathrm{H}]^{+}$(calcd for $\mathrm{C}_{19} \mathrm{H}_{23} \mathrm{~N}_{2} \mathrm{O}_{6} \mathrm{~S}_{2}, 439.0998$ ).

Eutypellazine G (7). Orthorhombic crystal; mp 204-206 ${ }^{\circ} \mathrm{C}$; $[\alpha]_{\mathrm{D}}^{25}+120\left(c\right.$ 0.5, MeOH); UV (MeOH) $\lambda_{\max }(\log \varepsilon) 204$ (4.19) nm; IR (KBr) $\nu_{\max } 3392,2923,1707,1653,1420,1324 \mathrm{~cm}^{-1} ;{ }^{1} \mathrm{H}$ and ${ }^{13} \mathrm{C}$ NMR data, see Tables 3 and 4; HRESIMS $m / z$ 441.1151 [M + $\mathrm{H}]^{+}$(calcd for $\mathrm{C}_{19} \mathrm{H}_{25} \mathrm{~N}_{2} \mathrm{O}_{6} \mathrm{~S}_{2}, 441.1154$ ).

Eutypellazine $\mathbf{H}$ (8). Orthorhombic crystal; mp 298-300 ${ }^{\circ} \mathrm{C}$; $[\alpha]_{\mathrm{D}}^{25}+134(c 0.2, \mathrm{MeOH})$; UV (MeOH) $\lambda_{\max }(\log \varepsilon) 206(4.16) \mathrm{nm}$; IR (KBr) $\nu_{\max } 3363,2923,1703,1648,1414,1241 \mathrm{~cm}^{-1} ;{ }^{1} \mathrm{H}$ and ${ }^{13} \mathrm{C}$ NMR data, see Tables 3 and 4; HRESIMS $\mathrm{m} / \mathrm{z}$ 439.1000 [M + $\mathrm{H}]^{+}$(calcd for $\mathrm{C}_{19} \mathrm{H}_{23} \mathrm{~N}_{2} \mathrm{O}_{6} \mathrm{~S}_{2}, 439.0998$ ).

Eutypellazine I (9). White powder; $[\alpha]_{\mathrm{D}}^{25}-94(c 0.1, \mathrm{MeOH})$; $\mathrm{UV}(\mathrm{MeOH}) \lambda_{\max }(\log \varepsilon) 202$ (4.09), 300 (3.74) nm; IR (KBr) $\nu_{\max }$

Table $2{ }^{1} \mathrm{H}$ NMR data of compounds $1-5$ in DMSO- $d_{6}$

\begin{tabular}{|c|c|c|c|c|c|}
\hline Position & 1 & 2 & 3 & 4 & 5 \\
\hline 3 & $\begin{array}{l}3.03, \mathrm{~d}(14.0) ; \\
3.05, \mathrm{~d}(14.0)\end{array}$ & $\begin{array}{l}\text { 3.01, d (14.0); } \\
3.04 \text { d (14.0) }\end{array}$ & $\begin{array}{l}3.01, \mathrm{~d}(14.0) \\
3.05(14.0)\end{array}$ & $\begin{array}{l}3.00 \text {, d (14.0); } \\
3.04 \text {, d (14.0) }\end{array}$ & $\begin{array}{l}\text { 2.98, d (15.5); } \\
3.06, \text { d (15.5) }\end{array}$ \\
\hline 6 & 5.91, dd $(10.0,4.5)$ & 5.90, dd $(10.0,4.5)$ & 5.91, dd $(9.8,4.5)$ & 5.89, dd $(9.8,4.5)$ & 5.91, dd $(9.8,4.5)$ \\
\hline 7 & 5.62, brd $(10.0)$ & $5.62, \mathrm{~d}(10.0)$ & 5.62, brd $(9.8)$ & 5.62, brd $(9.8)$ & 5.65, brd $(9.8)$ \\
\hline 8 & 4.66 , brd (13.8) & 4.65 , brd (13.9) & 4.60, brd $(13.6)$ & 4.58, brd $(13.3)$ & $4.63, \mathrm{~d}(13.8)$ \\
\hline & 2.98, dd $(13.1,8.5)$ & 2.79, dd $(13.0,1.5)$ & 3.07, dd $(11.6,7.0)$ & $2.83, \mathrm{~d}(13.2)$ & 2.44, dd $(12.7,5.0)$ \\
\hline $4^{\prime}$ & 3.03, dd $(11.5,8.0)$ & $2.69, \mathrm{dt}(8.5,8.0)$ & $3.16, \mathrm{t}(7.0)$ & 3.00, dd $(8.0,7.8)$ & $3.42, \mathrm{dt}(13.3,5.0)$ \\
\hline $5^{\prime}$ & & $4.31, \mathrm{~m}$ & & & \\
\hline $6^{\prime}$ & $2.92, \mathrm{dd}(18.8,7.5)$ & 1.97, dd $(14.6,7.8)$ & $3.64, \mathrm{~d}(8.9)$ & 2.24, ddd $(12.0,6.0,8.0)$ & $6.06, \mathrm{dd}(10.2,2.0)$ \\
\hline & $3.12, \mathrm{~d}(18.8)$ & $2.74, \mathrm{~m}$ & & $2.63, \mathrm{dt}(12.0,7.0)$ & \\
\hline $7^{\prime}$ & 3.74, dd $(7.5,5.8)$ & 3.39, dd $(8.2,3.8)$ & $2.53, \mathrm{dd}(15.4,8.9)$ & $1.92, \mathrm{~m}$ & $6.91, \mathrm{dd}(10.2,1.7)$ \\
\hline $\mathrm{CH}_{3} \mathrm{~S}-2^{\prime}$ & & & & $1.97, \mathrm{~s}$ & $2.17, \mathrm{~s}$ \\
\hline $\mathrm{OH}-8$ & $5.43, \mathrm{~d}(1.2)$ & $5.53, \mathrm{br}$ & $5.35, \mathrm{br}$ & $5.31, \mathrm{br}$ & $5.17, \mathrm{~d}(2.0)$ \\
\hline $\mathrm{OH}-5^{\prime}$ & & $4.85, \mathrm{~d}(4.6)$ & & & \\
\hline $\mathrm{OH}-8^{\prime}$ & $6.21, \mathrm{~d}(2.5)$ & 5.91, br & $5.56, \mathrm{br}$ & $5.37, \mathrm{br}$ & $6.18, \mathrm{br}$ \\
\hline
\end{tabular}


Table $3{ }^{1} \mathrm{H}$ NMR data of $6-13$ in DMSO- $d_{6}(400 \mathrm{MHz})$

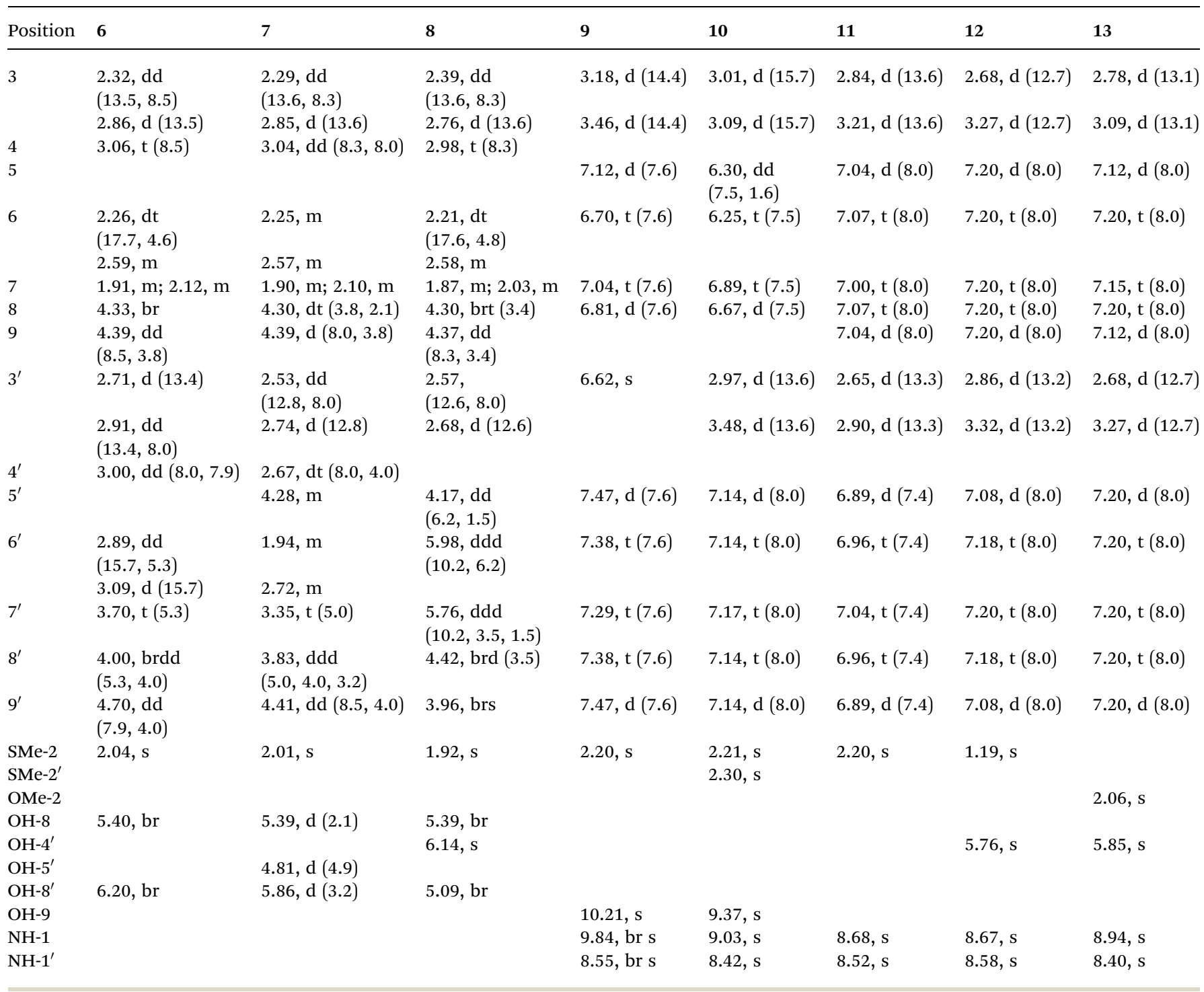

3734, 2921, 1670, 1448, 1417, 1375, $1237 \mathrm{~cm}^{-1} ;{ }^{1} \mathrm{H}$ and ${ }^{13} \mathrm{C}$ NMR data, see Tables 3 and 4; HRESIMS $m / z$ 355.1120 $[\mathrm{M}+\mathrm{H}]^{+}$(calcd for $\mathrm{C}_{19} \mathrm{H}_{19} \mathrm{~N}_{2} \mathrm{O}_{3} \mathrm{~S}, 355.1116$ ).

Eutypellazine J (10). White powder; $[\alpha]_{\mathrm{D}}^{25}-128(c 0.1, \mathrm{MeOH})$; $\mathrm{UV}(\mathrm{MeOH}) \lambda_{\max }(\log \varepsilon) 203$ (4.19), 276 (2.83) nm; IR (KBr) $\nu_{\max }$ $3180,2921,1838,1663,1847,1435,1369,1228 \mathrm{~cm}^{-1} ;{ }^{1} \mathrm{H}$ and ${ }^{13} \mathrm{C}$ NMR data, see Tables 3 and 4; HRESIMS $m / z$ 425.0965 [M + Na $]^{+}$ (calcd for $\mathrm{C}_{20} \mathrm{H}_{22} \mathrm{~N}_{2} \mathrm{O}_{3} \mathrm{~S}_{2} \mathrm{Na}, 425.0970$ ).

Eutypellazine K (11). White powder; $[\alpha]_{\mathrm{D}}^{25}-165$ (c 0.05, $\mathrm{MeOH}) ; \mathrm{UV}(\mathrm{MeOH}) \lambda_{\max }(\log \varepsilon) 202$ (4.11) nm; IR (KBr) $\nu_{\max }$ 3412, 3292, 3207, 1671, 1495, 1436, 1242, $1109 \mathrm{~cm}^{-1} ;{ }^{1} \mathrm{H}$ and ${ }^{13} \mathrm{C}$ NMR data, see Tables 3 and 4; HRESIMS $m / z$ 355.1112 $[\mathrm{M}-\mathrm{H}]^{-}$ (calcd for $\mathrm{C}_{19} \mathrm{H}_{19} \mathrm{~N}_{2} \mathrm{O}_{3} \mathrm{~S}, 355.1116$ ).

Eutypellazine L (12). White powder; $[\alpha]_{\mathrm{D}}^{25}-80$ (c 0.02, $\mathrm{MeOH})$; UV (MeOH) $\lambda_{\max }(\log \varepsilon) 202$ (4.01) nm; IR (KBr) $\nu_{\text {max }}$ 3275, 3204, 1672, 1496, 1453, 1437, 1403, 1307, $1240 \mathrm{~cm}^{-1} ;{ }^{1} \mathrm{H}$ and ${ }^{13} \mathrm{C}$ NMR data, see Tables 3 and 4; HRESIMS $m / z 355.1120$ $[\mathrm{M}-\mathrm{H}]^{-}$(calcd for $\mathrm{C}_{19} \mathrm{H}_{19} \mathrm{~N}_{2} \mathrm{O}_{3} \mathrm{~S}, 355.1116$ ).
Eutypellazine $\mathbf{M}$ (13). White powder; $[\alpha]_{\mathrm{D}}^{25}-72\left(\begin{array}{cl}c & 0.05\end{array}\right.$ $\mathrm{MeOH}) ; \mathrm{UV}(\mathrm{MeOH}) \lambda_{\max }(\log \varepsilon) 205$ (4.08) nm; IR (KBr) $\nu_{\max }$ $3430,3289,3206,1668,1495,1435,1405,1110 \mathrm{~cm}^{-1} ;{ }^{1} \mathrm{H}$ and ${ }^{13} \mathrm{C}$ NMR data, see Tables 3 and 4; HRESIMS $m / z$ 339.1342 $[\mathrm{M}-\mathrm{H}]^{-}$ (calcd for $\mathrm{C}_{19} \mathrm{H}_{19} \mathrm{~N}_{2} \mathrm{O}_{4}, 339.1345$ ).

\subsection{Preparation of $(R)$-MPA and $(S)$-MPA esters of 4}

Compound 4 (2.0 $\mathrm{mg}$ for each) was dissolved in $\mathrm{CHCl}_{3}(600 \mu \mathrm{L})$ and $(R)$-MPA $(3.0 \mathrm{mg})$, DMAP $(2.8 \mathrm{mg})$ and DCC $(2.5 \mathrm{mg})$ were added. The mixture was kept at room temperature for $12 \mathrm{~h}$. The reaction products were purified by silica gel CC eluted with $\mathrm{PE}$ : acetone $(5: 1)$ to get bis- $(R)$-MPA ester $\mathbf{4 a}(1.8 \mathrm{mg})$. In an identical protocol, bis- $(S)$-MPA ester of $\mathbf{4 b}(2.1 \mathrm{mg})$ was obtained from 4.

Bis-(R)-MPA ester of 4 (4a). ${ }^{1} \mathrm{H}$ NMR $\left(\mathrm{CDCl}_{3}, 400 \mathrm{MHz}\right) \delta_{\mathrm{H}}$ 7.34-7.47 (10H, m, phenyl protons), 5.93-5.97 (3H, m, H-5/H-6/ $\mathrm{H}-8), 5.76$ (1H, brs, H-8'), $5.58(1 \mathrm{H}, \mathrm{m}, \mathrm{H}-7), 5.31(1 \mathrm{H}, \mathrm{d}, J=$ $12.8 \mathrm{~Hz}, \mathrm{H}-9), 4.82$ (1H, s, CH of MPA), 4.79 (1H, s, CH of MPA), 
$\stackrel{\oplus}{\rightarrow}$

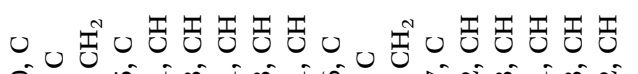

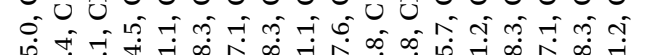

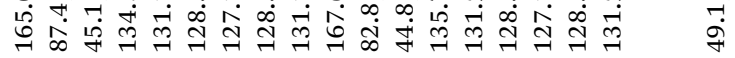

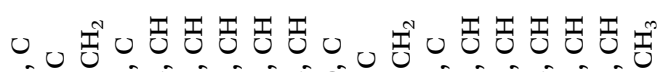
-

I $\quad \begin{aligned} & 0 \\ & 0\end{aligned}$

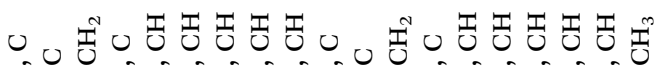

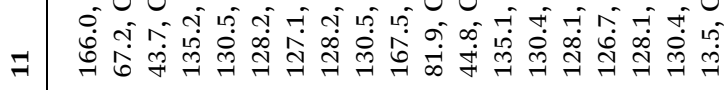

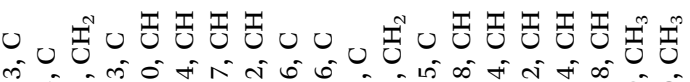

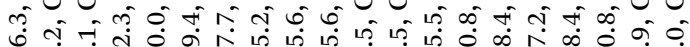

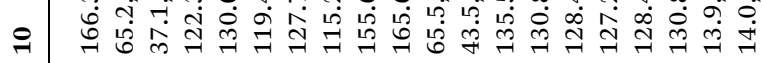

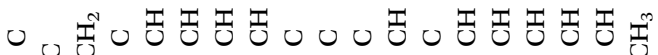

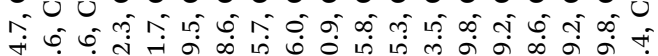

$a$

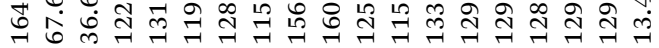

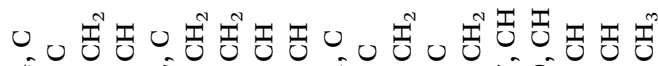

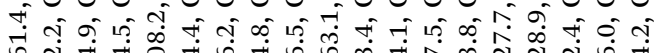

$\infty$

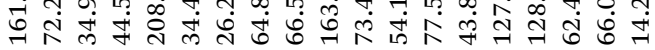

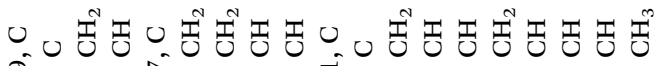

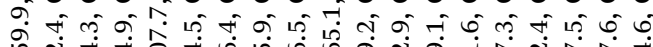

$\wedge$

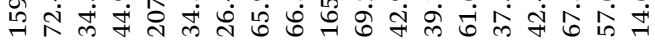

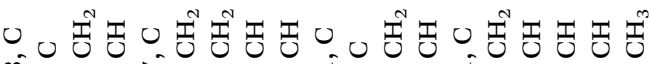

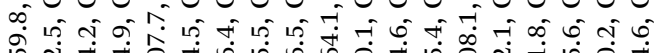

$\bullet$

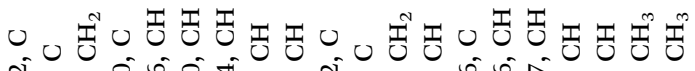
ภู

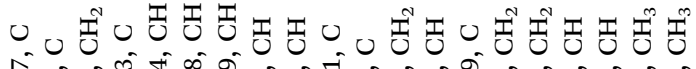

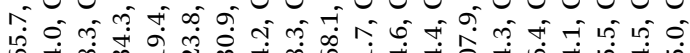

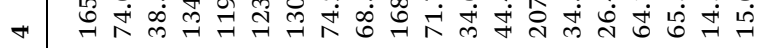

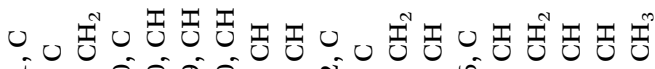

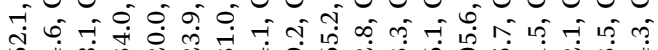

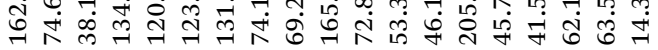

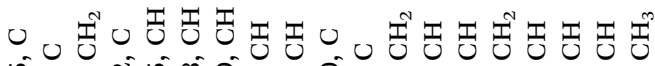

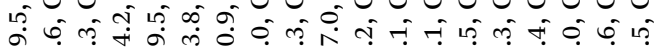

ง मि

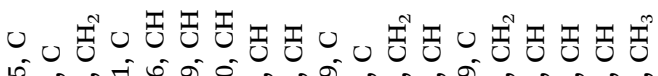
L

- मी 
$4.45\left(1 \mathrm{H}, \mathrm{d}, J=7.8 \mathrm{~Hz}, \mathrm{H}-9^{\prime}\right), 3.52$ (3H, OMe of MPA), $3.46(3 \mathrm{H}$, OMe of MPA), 3.07 (1H, d, $\left.J=13.9 \mathrm{~Hz}, \mathrm{H}-3^{\prime} \mathrm{a}\right), 3.02(1 \mathrm{H}, \mathrm{d}, J=$ 16.2 Hz, H-3a), 2.85 (1H, d, $J=16.2 \mathrm{~Hz}, \mathrm{H}-3 \mathrm{~b}), 2.75$ (1H, t, $J=$ $7.8 \mathrm{~Hz}, \mathrm{H}-4^{\prime}$ ), 2.32 (1H, m, H-7'a), 2.25 (1H, m, H-6'a), 2.21 (3H, s, SMe-2), 2.13 (1H, dd, $\left.J=7.8,14.1 \mathrm{~Hz}, \mathrm{H}-3^{\prime} \mathrm{b}\right), 2.02(1 \mathrm{H}, \mathrm{m}$, $\left.\mathrm{H}-7^{\prime} \mathrm{b}\right), 1.82$ (1H, m, H-6'b), 1.80 (3H, s, SMe-2').

Bis-(S)-MPA ester of 4 (4b). ${ }^{1} \mathrm{H}$ NMR $\left(\mathrm{CDCl}_{3}, 400 \mathrm{MHz}\right) \delta_{\mathrm{H}}$ 7.33-7.47 (10H, m, phenyl protons), 5.92-5.97 (3H, m, H-5/H-6/H8), $5.84(1 \mathrm{H}$, brs, H-8'), $5.58(1 \mathrm{H}, \mathrm{m}, \mathrm{H}-7), 5.27(1 \mathrm{H}, \mathrm{d}, J=13.2 \mathrm{~Hz}$, $\mathrm{H}-9), 4.82$ (1H, s, CH of MPA), $4.78(1 \mathrm{H}, \mathrm{s}, \mathrm{CH}$ of MPA), $4.23(1 \mathrm{H}, \mathrm{d}$, $J=7.6 \mathrm{~Hz}$ ), 3.51 (3H, OMe of MPA), 3.46 (3H, OMe of MPA), 3.01 $\left(1 \mathrm{H}, \mathrm{d}, J=14.1 \mathrm{~Hz}, \mathrm{H}-3^{\prime} \mathrm{a}\right), 3.00(1 \mathrm{H}, \mathrm{d}, J=16.1 \mathrm{~Hz}, \mathrm{H}-3 \mathrm{a}), 2.82$ $(1 \mathrm{H}, \mathrm{d}, J=16.1 \mathrm{~Hz}, \mathrm{H}-3 \mathrm{~b}), 2.47$ (1H, m, H-7'a), $2.43\left(2 \mathrm{H}, \mathrm{m}, \mathrm{H}-4^{\prime}\right.$ / H-6'a), 2.36 (1H, m, H-6'b), 2.19 (3H, s, SMe-2), 2.18 (1H, m, H$\left.7^{\prime} \mathrm{b}\right), 2.04$ (1H, dd, $\left.J=7.8,14.1 \mathrm{~Hz}, \mathrm{H}-3^{\prime} \mathrm{b}\right), 1.77$ (3H, s, SMe-2').

\subsection{X-ray single crystal diffraction}

Eutypellazine A (1) was obtained colorless crystal from $\mathrm{MeOH} /$ $\mathrm{H}_{2} \mathrm{O}(50: 1)$ using the vapor diffusion method. The monoclinic crystal $(0.06 \times 0.04 \times 0.03)$ was measured on Bruker D8 Advance single crystal X-ray diffractometer with $\mathrm{Cu}-\mathrm{K} \alpha$ radiation at $104.7 \mathrm{~K}$. Crystal data of 1 : empirical formula $\mathrm{C}_{19} \mathrm{H}_{22} \mathrm{~N}_{2} \mathrm{O}_{6} \mathrm{~S}_{2}, M$ $=438.51$; space group $P 2_{1}$, unit cell dimensions $a=7.6255$ (4) $\mathrm{A}$, $b=27.2984(13) \AA, c=9.2094(5) \AA, \alpha=\gamma=90.00^{\circ}, \beta=$ 90.415(5) ${ }^{\circ}, V=1917.01(17) \AA, Z=4, D_{\text {calcd }}=1.1519 \mathrm{mg} \mathrm{m}^{-3}, \mu$ $=2.886 \mathrm{~mm}^{-1}, F(000)=920$; a total of 13001 reflections were collected in the range of $6.48^{\circ}<2 \theta<142.48^{\circ}$, of which 7261 independent reflections $\left[R_{(\mathrm{int})}=0.0486\right.$ (inf- $0.9 \AA$ A) $]$ were used for the analysis. The structure was solved by the direct methods with the SHELXL-97 program and refined using full-matrix least-squares difference Fourier techniques. The final $R$ indexes [all data] gave $R_{1}=0.0484, \mathrm{w} R_{2}=0.1142$ and the Flack parameter $=-0.004(14)$. Crystallographic data of 1 have been deposited in the Cambridge Crystallographic Data Center (deposition number CCDC 1416589†).

Eutypellazine E (5) was obtained colorless crystal from $\mathrm{MeOH}$ using the vapor diffusion method. The orthorhombic crystal $(0.30 \times 0.25 \times 0.05)$ was measured on Bruker D8 Advance single crystal $\mathrm{X}$-ray diffractometer with $\mathrm{Cu}-\mathrm{K} \alpha$ radiation at 99.9 K. Crystal data of 5: empirical formula $\mathrm{C}_{20.21} \mathrm{H}_{22.84} \mathrm{~N}_{2} \mathrm{O}_{5.40} \mathrm{~S}_{2}, M=444.37$; space group $P 2{ }_{1} 2_{1} 2_{1}$, unit cell dimensions $a=14.08776(18) \AA, b=15.8916(19) \AA, c=$ 27.9520(4) $\AA$, $\alpha=\beta=\gamma=90.00^{\circ}, V=6257.82(14) \AA, Z=12$, $D_{\text {calcd }}=1.1415 \mathrm{mg} \mathrm{m}^{-3}, \mu=2.639 \mathrm{~mm}^{-1}, F(000)=2800$; a total of 42403 reflections were collected in the range of $6.40^{\circ}<2 \theta<$ $142.14^{\circ}$, of which 11963 independent reflections $\left[R_{(\mathrm{int})}=0.0396\right.$ (inf-0.9 ̊)] were used for the analysis. The structure was solved by the direct methods with the SHELXL-97 program and refined using full-matrix least-squares difference Fourier techniques. The final $R$ indexes [all data] gave $R_{1}=0.0463, \mathrm{w} R_{2}=0.1175$ and the Flack parameter $=0.015(12)$. Crystallographic data of 5 have been deposited in the Cambridge Crystallographic Data Center (deposition number CCDC 1416590†).

Upon crystallization from $\mathrm{MeOH}-\mathrm{H}_{2} \mathrm{O}$ (100:1) using the vapor diffusion method, colorless crystals were obtained for eutypellazine F (6). The orthorhombic crystal $(0.40 \times 0.35 \times$
0.25) was measured on Bruker D8 Advance single crystal X-ray diffractometer with $\mathrm{Cu}-\mathrm{K} \alpha$ radiation at $101.8 \mathrm{~K}$. Crystal data of 6: empirical formula $\mathrm{C}_{19} \mathrm{H}_{24} \mathrm{~N}_{2} \mathrm{O}_{7} \mathrm{~S}_{2}, M=456.52$; space group $P 2{ }_{1} 2_{1} 2{ }_{1}$, unit cell dimensions $a=9.5541(9) \AA, b=13.1555(17) \AA$, $c=15.7112(6) \AA, \alpha=\beta=\gamma=90.00^{\circ}, V=1974.7(3) \AA ㅇ, ~ Z=4$, $D_{\text {calcd }}=1.536 \mathrm{mg} \mathrm{m}^{-3}, \mu=2.864 \mathrm{~mm}^{-1}, F(000)=960$; a total of 6784 reflections were collected in the range of $8.76^{\circ}<2 \theta<$ $141.64^{\circ}$, of which 3711 independent reflections $\left[R_{\text {(int) }}=0.0199\right.$ (inf-0.9 A)] were used for the analysis. The structure was solved by the direct methods with the SHELXL-97 program and refined using full-matrix least-squares difference Fourier techniques. The final $R$ indexes [all data] gave $R_{1}=0.0300, \mathrm{w} R_{2}=0.0772$ and the Flack parameter $=0.007(13)$. Crystallographic data of 6 have been deposited in the Cambridge Crystallographic Data Center (deposition number CCDC 1416591†).

Eutypellazine $\mathrm{G}$ (7) was obtained colorless crystal from $\mathrm{MeOH}-\mathrm{H}_{2} \mathrm{O}$ (50:1) using the vapor diffusion method. The monoclinic crystal $(0.15 \times 0.15 \times 0.10)$ was measured on Bruker D8 Advance single crystal X-ray diffractometer with $\mathrm{Cu}-\mathrm{K} \alpha$ radiation at $103.3 \mathrm{~K}$. Crystal data of 7 : empirical formula $\mathrm{C}_{20} \mathrm{H}_{30} \mathrm{~N}_{2} \mathrm{O}_{8} \mathrm{~S}_{2}, M=490.58$; space group $P 2_{1} 2_{1} 2_{1}$, unit cell dimensions $a=9.16165(19) \AA, b=9.5804(2) \AA, c=25.5833(6) \AA$, $\alpha=\beta=\gamma=90.00^{\circ}, V=2245.52(9) \AA, Z=4, D_{\text {calcd }}=1.451 \mathrm{mg}$ $\mathrm{m}^{-3}, \mu=2.589 \mathrm{~mm}^{-1}, F(000)=1040$; a total of 7719 reflections were collected in the range of $6.92^{\circ}<2 \theta<142.36^{\circ}$, of which 4256 independent reflections $\left[R_{\text {(int) }}=0.0280\right.$ (inf- $0.9 \AA$ ) $]$ were used for the analysis. The structure was solved by the direct methods with the SHELXL-97 program and refined using full-matrix least-squares difference Fourier techniques. The final $R$ indexes [all data] gave $R_{1}=0.0414, \mathrm{w} R_{2}=0.1015$ and the Flack parameter $=-0.003(18)$. Crystallographic data of 7 have been deposited in the Cambridge Crystallographic Data Center (deposition number CCDC 1416592 $\dagger$ ).

Eutypellazine $\mathrm{H}$ (8) was obtained colorless crystal from $\mathrm{MeOH}$ using the vapor diffusion method. The orthorhombic crystal $(0.60 \times 0.25 \times 0.25)$ was measured on Bruker D8 Advance single crystal $\mathrm{X}$-ray diffractometer with $\mathrm{Cu}-\mathrm{K} \alpha$ radiation at $102.4 \mathrm{~K}$. Crystal data of 8: empirical formula $\mathrm{C}_{19} \mathrm{H}_{22} \mathrm{~N}_{2} \mathrm{O}_{6} \mathrm{~S}_{2}, M$ $=438.51$; space group $P 2{ }_{1} 2_{1} 2_{1}$, unit cell dimensions $a=$ 8.27744(17) ̊, $b=10.60684(20) \AA, c=21.3739(4) \AA, \alpha=\beta=\gamma=$ $90.00^{\circ}, V=1876.57(6) \AA, Z=4, D_{\text {calcd }}=1.552 \mathrm{mg} \mathrm{m}^{-3}, \mu=2.948$ $\mathrm{mm}^{-1}, F(000)=920$; a total of 7441 reflections were collected in the range of $8.28^{\circ}<2 \theta<142.24^{\circ}$, of which 3517 independent reflections $\left[R_{(\mathrm{int})}=0.0238\right.$ (inf-0.9 $\AA$ ) $]$ were used for the analysis. The structure was solved by the direct methods with the SHELXL-97 program and refined using full-matrix least-squares difference Fourier techniques. The final $R$ indexes [all data] gave $R_{1}=0.0330, \mathrm{w} R_{2}=0.0827$ and the Flack parameter $=$ $-0.008(13)$. Crystallographic data of 8 have been deposited in the Cambridge Crystallographic Data Center (deposition number CCDC 1416593†).

\subsection{Anti-HIV bioassay}

$293 \mathrm{~T}$ cells were cultured at $37{ }^{\circ} \mathrm{C}$ in a $5 \% \mathrm{CO}_{2}$ humidified atmosphere and split twice a week. The vector pNL4.3-Luc was generated by cloning the luciferase gene (Thermo Fisher 
Scientific) in the HIV-1 proviral clone pNL4.3. Plasmid pNL4.3Ren was generated by cloning the renilla gene (Promega) in the Luc site of pNL4.3-Luc (NIH AIDS Reagent Program). Infectious supernatants were obtained from $\mathrm{Ca}_{3}(\mathrm{PO} 4)_{2}$ transfection on 293T cells of plasmid pNL4.3-Ren. These supernatants were used to infect cells in the presence or absence of the compounds to be evaluated. Anti-HIV activity quantification was performed $48 \mathrm{~h}$ postinfection. Briefly, cells were lysed with $100 \mu \mathrm{L}$ of buffer. Relative luminescence units (RLUs) were obtained in a luminometer (Berthold Detection Systems) after the addition of substrate to cell extracts. Viability was performed in parallel treated cells with the same concentrations of compound. After 48 h, cell viability was evaluated with the CellTiter Glo (Promega) assay system following the manufacturer's specifications. Inhibitory concentrations $50 \%\left(\mathrm{IC}_{50}\right)$ and cytotoxic concentrations $50 \%\left(\mathrm{CC}_{50}\right)$ were calculated using GraphPad Prism software.

\subsection{In vitro latent HIV reactivating assay}

For flow cytometry-bases screening, the J-Lat A2 cell line containing an integrated HIV-1 long terminal repeat (LTR) luciferase reporter construct but expressing no Tat was used. The JLat A2 cells were grown in Dulbecco's modification of eagle's medium (DMEM) (Thermo Fisher Scientific) supplemented with $10 \%$ fetal bovine serum (Aleken Biologicals), $2 \mathrm{mM} \mathrm{L}^{-}$ glutamine (Invitrogen), $4500 \mathrm{mg} \mathrm{mL}^{-1}$ glucose, and antibiotic solution (0.2\% kanamycin, $0.2 \%$ streptomycin, and $0.12 \%$ penicillin) (Sigma Aldrich) at $37{ }^{\circ} \mathrm{C}$ under $5 \% \mathrm{CO}_{2}$ atmosphere. The DMEM replaced every date until cells grew to $90 \%$ confluency from explants and then using Tyrisin (Invitrogen) to dissociate them. These cultures of J-Lat A2 cells were then dispensed into 24 -well plates at $1 \times 10^{5}$ cells per $\mathrm{mL}$ per well $24 \mathrm{~h}$ prior to the test compounds treatment and incubated at $37^{\circ} \mathrm{C}$ under $5 \% \mathrm{CO}_{2}$ condition. The test compounds $(100 \mu \mathrm{M}$ for each), two positive controls prostratin $(5 \mu \mathrm{M})$, and SAHA (2.5 $\mu \mathrm{M})$, and negative control DMSO $(10 \mu \mathrm{L})$ were added to per well. After $48 \mathrm{~h}$ incubation, cells were rinsed and resuspended by phosphate-buffered saline (PBS) (Thermo Fisher Scientific) and then the flow cytometry was used to detect EGFP-positive cells. Cytotoxicities of all compounds were measured by the Promega CellTiter kit (CellTiter-Glo® Reagent) following the manufacturer's instructions in 96-well plates incubated with test compounds at $37{ }^{\circ} \mathrm{C}$ for $48 \mathrm{~h}$.

\section{Results and discussion}

\subsection{Structure elucidation of new compounds}

The EtOAc extract of the fermentation broth of Eutypella sp. MCCC 3A00281 was examined by the HPLC-ESIMS, which featured an array of TDKP-based derivatives. Detailed chromatographic separation including semipreparative HPLC purification resulted in the isolation of 15 TDKP analogues including 13 new compounds (Fig. 1).

Eutypellazine A (1) was isolated as white monoclinic crystals. Its molecular formula was established as $\mathrm{C}_{19} \mathrm{H}_{20} \mathrm{~N}_{2} \mathrm{O}_{5} \mathrm{~S}_{2}$ by the HRESIMS $\left(m / z 421.0887[\mathbf{M}+\mathbf{H}]^{+}\right)$and NMR data. The IR absorptions at 3370, 1713 and $1648 \mathrm{~cm}^{-1}$ suggested the presence of hydroxy and carbonyl functionalities. The ${ }^{1} \mathrm{H}$ and ${ }^{13} \mathrm{C}$ NMR data (Tables 1 and 2) were characteristic of a diketopiperazine-based derivative, while analyses of ${ }^{1} \mathrm{H}-{ }^{1} \mathrm{H}$ COSY, HSQC and HSBC data revealed the presence of a 6/5/6/5/6membered pentacyclic diketopiperazine skeleton, structurally related to the coexisted epicoccin $\mathrm{I}^{\mathbf{1 5}}$ The spin system coupled the protons from the olefinic proton $\mathrm{H}-5\left(\delta_{\mathrm{H}} 5.98\right)$ to $\mathrm{H}-9\left(\delta_{\mathrm{H}}\right.$ $4.78)$, while the HMBC correlations from $\mathrm{H}-9$ to C-2 $\left(\delta_{\mathrm{C}} 74.7\right)$, C$3\left(\delta_{\mathrm{C}} 38.3\right), \mathrm{C}-4\left(\delta_{\mathrm{C}} 134.1\right)$, and $\mathrm{C}-5\left(\delta_{\mathrm{C}} 119.6\right)$ and from $\mathrm{H}-5$ to $\mathrm{C}-3$ and $\mathrm{C}-9\left(\delta_{\mathrm{C}} 69.3\right)$ assigned a dihydroindoline for rings $\mathrm{A}-\mathrm{B}$, in which a hydroxy substitution at C-8 $\left(\delta_{\mathrm{C}} 73.9\right)$ was deduced by the COSY relationship between $\mathrm{H}-8\left(\delta_{\mathrm{H}} 4.66\right)$ and a $\mathrm{D}_{2} \mathrm{O}$ exchangeable proton $\mathrm{OH}-8\left(\delta_{\mathrm{H}} 5.43\right)$. Extensive analyses of the $2 \mathrm{D} \mathrm{NMR}$ data uncovered rings $\mathrm{D}-\mathrm{E}$ to be a perhydroindole, in which the location of a ketone group at C-5 $5^{\prime}\left(\delta_{\mathrm{C}} 207.9\right)$ and a hydroxy group at $\mathrm{C}-8^{\prime}\left(\delta_{\mathrm{C}} 66.1\right)$ was evident from the HMBC correlations from $\mathrm{C}-5^{\prime}$ to $\mathrm{H}_{2}-3^{\prime}, \mathrm{H}-4^{\prime}$, and $\mathrm{H}_{2}-6^{\prime}$ and the COSY relationship between $\mathrm{H}-8^{\prime}\left(\delta_{\mathrm{H}} 4.03\right)$ and $\mathrm{OH}-8^{\prime}\left(\delta_{\mathrm{H}} 6.21\right)$. In addition, the chemical shifts of a methyl group at $\delta_{\mathrm{H}} 2.24(3 \mathrm{H}, \mathrm{s}) / \delta_{\mathrm{C}} 14.5$ were characteristic of a thiomethyl group, which was located at C- 2 on the basis of the HMBC correlation between the methyl protons and $\mathrm{C}-2$. The second sulfur element was bonded across C-2 $\left(\delta_{\mathrm{C}} 70.1\right)$ and $\mathrm{C}-7^{\prime}\left(\delta_{\mathrm{C}} 41.8\right)$ to form a thioether bridge on the basis of the HMBC correlation between $\mathrm{H}-7^{\prime}\left(\delta_{\mathrm{H}} 3.74\right)$ and $\mathrm{C}-2^{\prime}$.

The relative configurations of $\mathbf{1}$ were established by the coupling constants and the NOE data. The $J_{\mathrm{H}-8 / \mathrm{H}-9}$ value $(13.8$ $\mathrm{Hz}$ ) in association with the NOE interaction between $\mathrm{OH}-8$ and $\mathrm{H}-9$ was indicative of trans orientation of $\mathrm{H}-8$ toward $\mathrm{H}-9$. The observation of the NOE correlations from $\mathrm{CH}_{3} \mathrm{~S}\left(\delta_{\mathrm{H}} 2.24\right)$ to $\mathrm{H}-8$ and $\mathrm{H}-8^{\prime}$, and from $\mathrm{H}-\mathrm{9}^{\prime}$ to $\mathrm{OH}-8^{\prime}$ and $\mathrm{H}-4^{\prime}$ assigned the same face of thiomethyl group as $\mathrm{H}-8$ and $\mathrm{H}-8^{\prime}$, while the cis fusion of rings $\mathrm{D}$ and $\mathrm{E}$ was deduced by the NOE interactions from $\mathrm{H}-4^{\prime}$ and $\mathrm{H}-\mathrm{9}^{\prime}$ to $\mathrm{OH}-\mathrm{8}^{\prime}$. The thioether orientation in opposite face to $\mathrm{H}-9^{\prime}$ was due to the NOE interaction between $\mathrm{OH}-\mathrm{s}^{\prime}$ and $\mathrm{H}-\mathrm{7}^{\prime}$ (Fig. 2). The absolute configurations of the stereogenic centers were determined by the X-ray diffraction experiment, while the Flack parameter $(-0.004(14))$ using $\mathrm{Cu}-\mathrm{K} \alpha$ reflection measurement unambiguously determined $2 R, 8 S, 9 S, 2^{\prime} R, 4^{\prime} R, 7^{\prime} R, 8^{\prime} R$, and $9^{\prime} S$ configurations, respectively (Fig. 3).

Analyses of the 2D NMR data revealed the structure of eutypellazine $\mathrm{B}$ (2) closely related to 1 . The distinction was observed in ring $\mathrm{E}$, where a ketone at $\mathrm{C}-5^{\prime}$ of $\mathbf{1}$ to be replaced by a hydroxy group, as recognized by the COSY relationship between the $\mathrm{D}_{2} \mathrm{O}$ exchangeable proton at $\delta_{\mathrm{H}} 4.85(\mathrm{OH})$ and $\mathrm{H}-5^{\prime}$ $\left(\delta_{\mathrm{H}} 4.31\right)$, in addition to the $\mathrm{HMBC}$ correlations from the $\mathrm{OH}$ proton to $\mathrm{C}-4^{\prime}, \mathrm{C}-5^{\prime}\left(\delta_{\mathrm{C}} 61.5\right)$, and C- $6^{\prime}$. Comparison of the NOE data and coupling constants indicated that both 2 and 1 share the same relative configurations in rings A-C, whereas the NOE interaction between $\mathrm{H}-5^{\prime} / \mathrm{H}-9^{\prime}$ and $\mathrm{OH}-5^{\prime} / \mathrm{H}-8^{\prime}$ (Fig. 2) assigned the opposite face of $\mathrm{OH}-5^{\prime}$ and $\mathrm{OH}-8^{\prime}$. Considering the absolute configuration established for 1 by X-ray data, the similar ECD data such as the positive Cotton effects at 230 and $270 \mathrm{~nm}$ which reflected the orientation of the thiomethyl group and thioether assumed both 2 and 1 sharing the same absolute configurations. Thus, the stereogenic center $\mathrm{C}-5^{\prime}$ in 2 was suggested to have the $S$ configuration. 

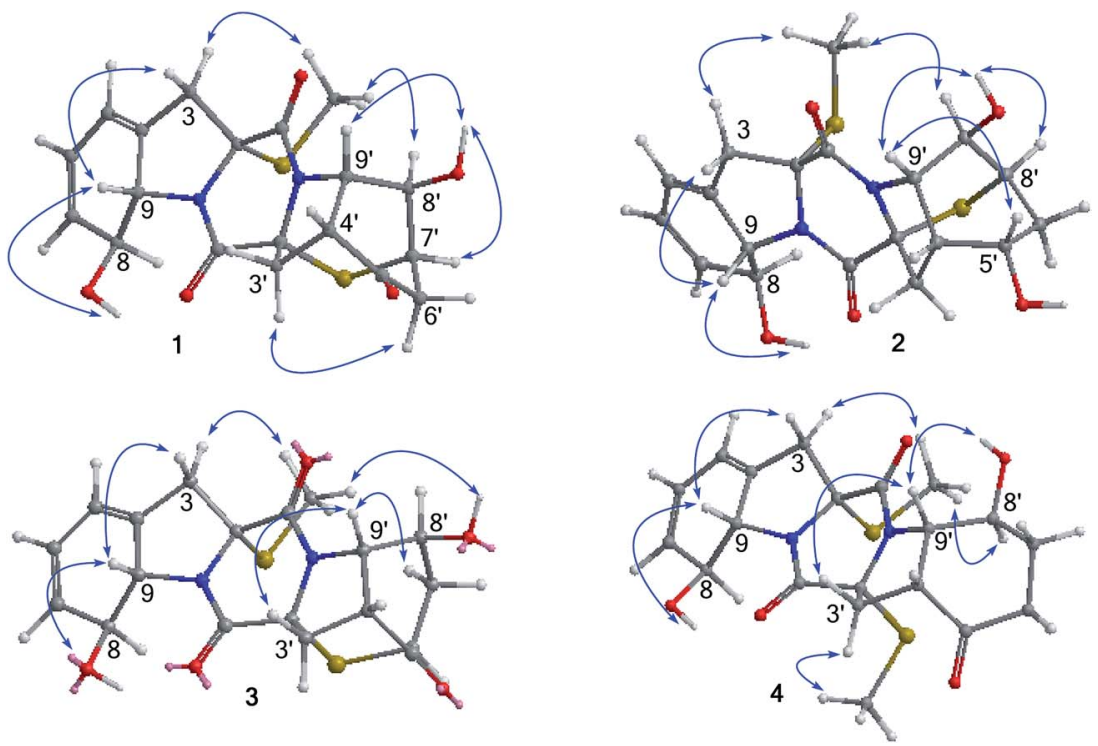

Fig. 2 Key NOE correlations of 1-4.
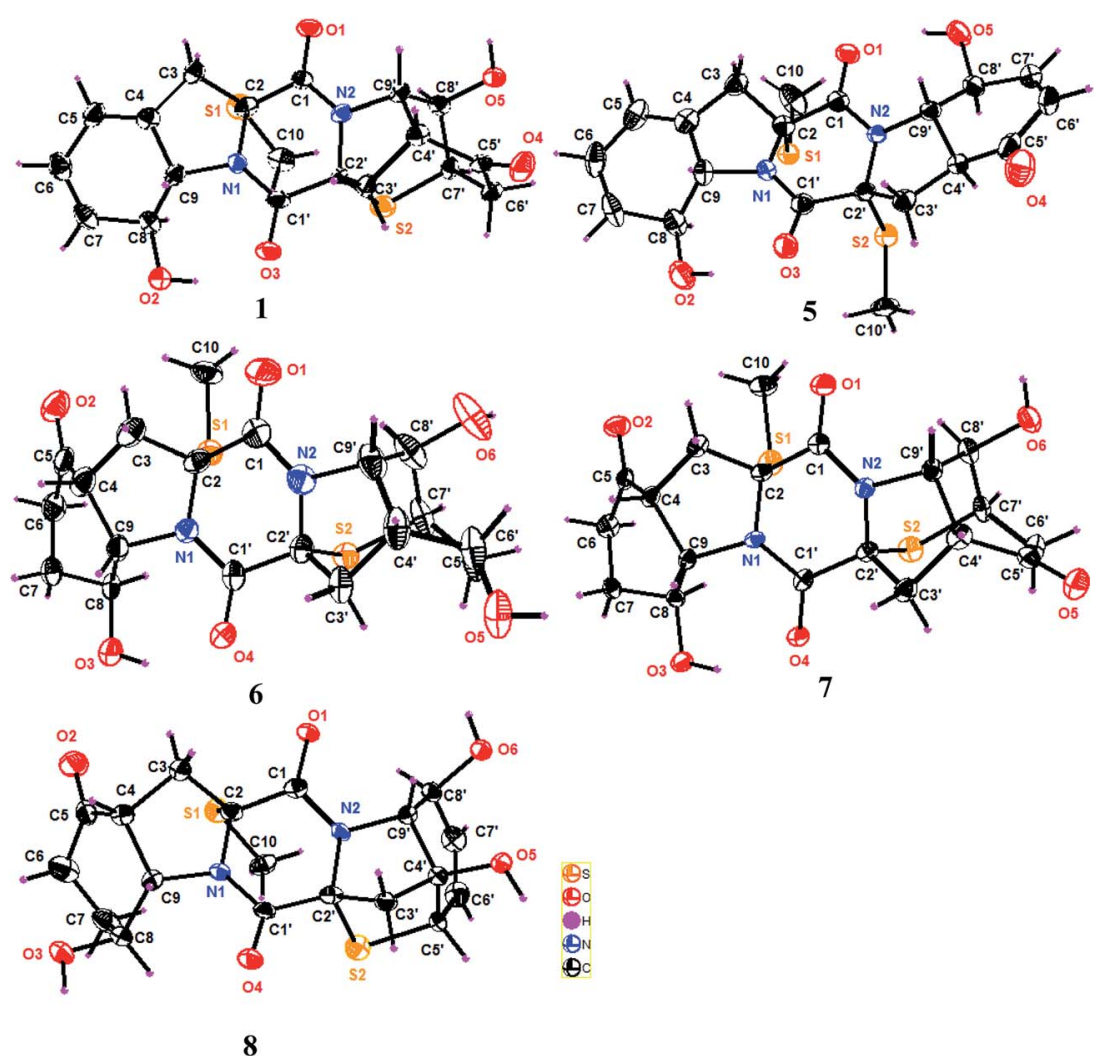

8

Fig. 3 ORTEP plots of the X-ray crystal structures of 1 and 5-8.

Eutypellazine C (3) has the same molecular formula as that of $\mathbf{1}$, as determined by the HRESIMS and NMR data. The 2D NMR data indicated that the partial structure of 3 regarding rings $\mathrm{A}-\mathrm{C}$ was identical to that of $\mathbf{1}$, while rings $\mathrm{D}$ and $\mathrm{E}$ presented as a perhydroindoline unit related to that of 1 . The COSY and HMQC data conducted $\mathrm{H}-6^{\prime}\left(\delta_{\mathrm{H}} 3.64\right)$ to be a methine proton instead of $\mathrm{H}-7^{\prime}$ ring $\mathrm{E}$ of $\mathbf{1}$. This assignment was evident from the COSY relationship from $\mathrm{H}_{2}-7^{\prime}\left(\delta_{\mathrm{H}} 2.53,2.69\right)$ to $\mathrm{H}-6^{\prime}$ and $\mathrm{H}-8^{\prime}\left(\delta_{\mathrm{H}} 4.50\right)$. The HMBC correlation between $\mathrm{H}-6^{\prime}$ and $\mathrm{C}-2^{\prime}$ confirmed the connection of a thioether bond across C- $2^{\prime}$ and C$6^{\prime}$. The closely similar NOE data (Fig. 2) and coupling constants allowed the assignment of the relative configurations of 3 to be 
the same as those of $\mathbf{1}$. The positive Cotton effects at 220 and $307 \mathrm{~nm}$ and the negative Cotton effect at $255 \mathrm{~nm}$ were attributed to $2 R$ configuration, whereas the positive Cotton effect at $270 \mathrm{~nm}$ was induced by the $\pi \rightarrow \pi^{*}$ transition of conjugated hexadiene. ${ }^{17}$ Thus, the remaining stereogenic centers of 3 were assigned as $2 R, 8 S, 9 S, 2^{\prime} R, 4^{\prime} R, 6^{\prime} S, 8^{\prime} S$, and $9^{\prime} S$, respectively.

The molecular formula of eutypellazine $\mathrm{D}(4)$ was established as $\mathrm{C}_{20} \mathrm{H}_{24} \mathrm{~N}_{2} \mathrm{O}_{5} \mathrm{~S}_{2}$ by the HRESIMS $\left(\mathrm{m} / z\right.$ 437.1201 $\left.[\mathrm{M}+\mathrm{H}]^{+}\right)$and NMR data. Comparison of the NMR (Tables 2 and 4) and ESIMS data indicated that the structure of $\mathbf{4}$ closely related to ent-epicoccin $\mathrm{G} .{ }^{15}$ The distinction was attributed to ring A, where the 2D NMR data assigned a 8-hydroxycyclohexadiene with the same moiety as that of 1 . Based on the modified Mosher's method, ${ }^{18}$ compound 4 was esterified by the $(R)$ - and $(S)$-MPA to form MPA esters $\mathbf{4 a}$ and $\mathbf{4 b}$. Calculation of the chemical shift values $\left(\Delta \delta^{R S}=\delta_{R}-\delta_{S}\right)$ resulted in a $S$ configuration for C-8 and C-8' (Fig. 4). In combination with the NOE interactions (Fig. 2), the absolute configurations of the remaining chiral centers were determined. These assignments were also supported by the negative Cotton effect at $262 \mathrm{~nm}$, which was in agreement with $2 R / 2^{\prime} R$ configurations for TDKPs bearing two $S$-methyl groups. ${ }^{17}$

Analyses of the 2D NMR data revealed that eutypellazine E (5) was a $6^{\prime}, 7^{\prime}$-dehydrogenated analogue of 4 . This assignment was evident from the similar NMR data of both $\mathbf{4}$ and $\mathbf{5}$, with the exception of the presence of two olefinic protons at $\delta_{\mathrm{H}} 6.06(\mathrm{H}-$ $\left.6^{\prime}\right)$ and $\delta_{\mathrm{H}} 6.91\left(\mathrm{H}-7^{\prime}\right)$ in addition to the HMBC correlations from $\mathrm{H}-6^{\prime}$ and $\mathrm{H}-\mathrm{7}^{\prime}$ to $\mathrm{C}-5^{\prime}$ and $\mathrm{C}-\mathrm{8}^{\prime}$. The NOE relationships revealed the same relative configuration in rings $\mathrm{A}-\mathrm{C}$ of both 4 and 5 . However, the NOE interactions between $\mathrm{H}-9^{\prime} / \mathrm{OH}-8^{\prime}$ and $\mathrm{H}-4^{\prime} / \mathrm{H}^{-}$ 8 , in association with $J_{\mathrm{H}-4^{\prime} / \mathrm{H}-9^{\prime}}$ value $(13.3 \mathrm{~Hz})$ conducted a trans fusion of rings $\mathrm{D}$ and $\mathrm{E}$. This assignment was further supported by the X-ray single crystal diffraction data using Flack parameter (0.015(12)) (Fig. 3), which deduced the absolute configurations to be $2 R, 8 S, 9 S, 2^{\prime} R, 4^{\prime} S, 8^{\prime} S$, and $9^{\prime} S$, respectively.

Eutypellazine $\mathrm{F}(6)$ has a molecular formula of $\mathrm{C}_{19} \mathrm{H}_{22} \mathrm{~N}_{2} \mathrm{O}_{6} \mathrm{~S}_{2}$ as determined by the HRESIMS and NMR data. Comparison of the NMR data in association with the 2D NMR data resulted in the partial structure regarding rings $\mathrm{C}-\mathrm{E}$ to be the same as that of 1 . The distinction was recognized in ring $\mathrm{A}$, where the location of a ketone group at C-5 was based on the COSY relationships of the spin system from $\mathrm{H}_{2}-3$ to $\mathrm{H}-9$, and the HMBC correlations from $\mathrm{C}-5$ to $\mathrm{H}_{2}-3, \mathrm{H}-4, \mathrm{H}_{2}-6, \mathrm{H}_{2}-7$, and $\mathrm{H}-9$. The
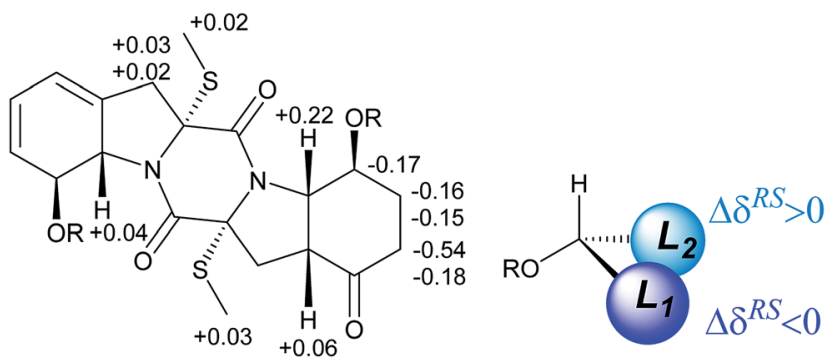

4a $\mathrm{R}=(R)-\mathrm{MPA}$ 4b $\mathrm{R}=(S)-\mathrm{MPA}$

Model for configurational correlation of MPA esters

Fig. $4 \Delta \delta^{R S}\left(\delta_{R}-\delta_{S}\right)$ values of the MPA esters of 4 in $\mathrm{CDCl}_{3}$. relative configurations of $\mathbf{6}$ were determined by the NOE relationships, while the absolute configurations were determined by the single crystal X-ray diffraction data using the Flack parameter $(0.007(13))$ as obtained by $\mathrm{Cu}-\mathrm{K} \alpha$ diffraction to assign $2 R, 4 R, 8 S, 9 S, 2^{\prime} R, 4^{\prime} R, 7^{\prime} R, 8^{\prime} R$, and $9^{\prime} S$, respectively (Fig. 3).

Comparison of the NMR data (Tables 3 and 4) indicated that the partial structure of rings A-C in eutypellazine $\mathrm{G}(7)$ is the same as that of $\mathbf{6}$, while the second partial structure in rings $\mathrm{C}-\mathrm{E}$ of 7 was identical to that of 2 . The absolute configurations of 7 were unequivocally assigned as $2 R, 4 R, 8 S, 9 S, 2^{\prime} R, 4^{\prime} R, 5^{\prime} S, 7^{\prime} R$, $8^{\prime} R$, and $9^{\prime} S$, respectively, on the basis of the Flack parameter $-0.003(18)$, which was obtained by the X-ray $\mathrm{Cu}-\mathrm{K} \alpha$ crystallographic experiment.

The 2D NMR data assigned eutypellazine $\mathrm{H}(\mathbf{8})$ to be a thiodiketopiperazine with the partial structure of rings $\mathrm{A}-\mathrm{C}$ being the same as that of 7, whereas the structure of rings C-E agreed with that of epicoccin I. ${ }^{15}$ The absolute configurations 8 were determined by the single crystal X-ray crystallographic data with the Flack parameter $-0.008(13)$, as obtained by the X-ray $\mathrm{Cu}-\mathrm{K} \alpha$ experiment, indicating $2 R, 4 R, 8 S, 9 S, 2^{\prime} R, 4^{\prime} S, 5^{\prime} S, 8^{\prime} S$, and $9^{\prime} R$, respectively.

The molecular formula of eutypellazine I (9) was established as $\mathrm{C}_{19} \mathrm{H}_{18} \mathrm{~N}_{2} \mathrm{O}_{3} \mathrm{~S}$ on the basis of the HRESIMS $(\mathrm{m} / \mathrm{z} 355.1120$, [M $+\mathrm{H}]^{+}$) and NMR data. The ${ }^{1} \mathrm{H}$ and ${ }^{13} \mathrm{C}$ NMR data (Tables 3 and 4 ) of 9 were closely related to those of coexisted emethacin $\mathrm{A},{ }^{\mathbf{1 9}}$ whereas the aromatic ring A presented a phenolic proton and an ABCD spin system instead of the mono-substituted aromatic ring of the known counterpart. The aromatic spin system among $\mathrm{H}-5\left(\delta_{\mathrm{H}} 7.12, \mathrm{~d}, J=7.3 \mathrm{~Hz}\right), \mathrm{H}-6\left(\delta_{\mathrm{H}} 6.70, \mathrm{t}, J=7.3 \mathrm{~Hz}\right), \mathrm{H}-$ $7\left(\delta_{\mathrm{H}} 7.04, \mathrm{t}, J=7.5 \mathrm{~Hz}\right)$, and $\mathrm{H}-8\left(\delta_{\mathrm{H}} 6.80, \mathrm{~d}, J=8.0 \mathrm{~Hz}\right)$, in association with the HMBC correlations from $\mathrm{H}_{2}-3$ to $\mathrm{C}-9\left(\delta_{\mathrm{C}}\right.$ 156.0), clarified 9 to be a 9-hydroxyemethacin A. This assignment was supported by the negative sign and the similar value of the specific rotation of both 9 and emethacin A.

Analyses of the NMR and HRESIMS data conducted eutypellazine $\mathrm{J}(\mathbf{1 0})$ to be a 9-hydroxyemethacin $\mathrm{B}$, while the distinction was attributed to the aromatic ring A where an ABCD spin system among $\mathrm{H}-5\left(\delta_{\mathrm{H}} 6.30\right), \mathrm{H}-6\left(\delta_{\mathrm{H}} 6.25\right), \mathrm{H}-7\left(\delta_{\mathrm{H}} 6.89\right)$, and $\mathrm{H}-8\left(\delta_{\mathrm{H}} 6.67\right)$ and the HMBC correlations from $\mathrm{H}_{2}-3\left(\delta_{\mathrm{H}}\right.$ $3.01,3.09)$ to $\mathrm{C}-4\left(\delta_{\mathrm{C}} 122.3\right), \mathrm{C}-5\left(\delta_{\mathrm{C}} 130.0\right)$, and $\mathrm{C}-9\left(\delta_{\mathrm{C}} 155.6\right)$ were observed in the ${ }^{1} \mathrm{H}^{-1} \mathrm{H}$ COSY and HMBC spectra. The similar magnitude and the same sign of the specific rotation of $10\left([\alpha]_{\mathrm{D}}^{25}-128, \mathrm{MeOH}\right)$ and emethacin $\mathrm{B}\left([\alpha]_{\mathrm{D}}^{25}-168, \mathrm{CHCl}_{3}\right)^{19}$ assumed 10 possessing $2 R / 2^{\prime} R$ configurations.

Comparison of the NMR data (Tables 3 and 4) indicated the structure of eutypellazine $\mathrm{K}(\mathbf{1 1})$ to be closely related to emethacin B. The distinction was found by the absence of a thiomethyl group and the deshielded C-2' $\left(\delta_{\mathrm{C}} 81.9\right)$ in the NMR spectra of 11. In addition, a $\mathrm{D}_{2} \mathrm{O}$ exchangeable proton $\left(\delta_{\mathrm{H}} 6.60\right.$, s) showed the HMBC correlations with $\mathrm{C}-1^{\prime}\left(\delta_{\mathrm{C}} 167.5\right), \mathrm{C}-2^{\prime}$, and C-3' $\left(\delta_{\mathrm{C}} 44.8\right)$, confirming $\mathrm{C}-2^{\prime}$ of 11 to be substituted by a hydroxy group to replace a thiomethyl group of emethacin $\mathrm{B}$. The NOE interaction between $\mathrm{CH}_{3} \mathrm{~S}$ and $\mathrm{OH}-2^{\prime}$ assigned the spatial approximation of both functional groups. In addition, the negative specific rotation of $11\left([\alpha]_{\mathrm{D}}^{25}-165, \mathrm{MeOH}\right)$ which was contributed by the chiral centers at C-2 and C-2' and was comparable to that of $\mathbf{1 0}$, suggested $2 R / 2^{\prime} R$ configurations. 
Eutypellazine L (12) was determined to have a planer structure to be the same as that of 11, based on the 2D NMR and HRESIMS data. The distinction was observed by the deshielded $\mathrm{C}-2^{\prime}\left(\delta_{\mathrm{C}} 82.7\right)$ and the lower magnitude of the specific rotation $\left([\alpha]_{\mathrm{D}}^{25}-80\right)$ in comparison with those of 11. Since the hydroxylated and methoxylated diatretol with $2 S$ and 2 'S configurations exhibited positive specific rotation $\left([\alpha]_{\mathrm{D}}^{25}+42, \mathrm{MeOH}\right),{ }^{20}$ the lowering value of the specific rotation of $\mathbf{1 1}$ was derived by the $2^{\prime} S$ contribution.

Eutypellazine M (13) has a molecular formula of $\mathrm{C}_{19} \mathrm{H}_{20} \mathrm{~N}_{2} \mathrm{O}_{4}$ as provided by the HRESIMS $\left(\mathrm{m} / z 339.1342[\mathrm{M}-\mathbf{H}]^{-}\right)$and NMR data. Analyses of the NMR data revealed that 13 structurally related to $\mathbf{1 1}$ with the exception of the substitution at $\mathrm{C}-2$, in which a methoxy group $\left(\delta_{\mathrm{H}} 2.06 / \delta_{\mathrm{C}} 49.1\right)$ instead of a thiomethyl group was recognized to position at $\mathrm{C}-2$. This assignment was supported by the HMBC correlation between the methoxy protons and C-2 $\left(\delta_{\mathrm{C}} 87.4\right)$. The remarkable shielded protons of $\mathrm{MeO}\left(\delta_{\mathrm{H}} 2.06\right)$ was due to the location of the $\mathrm{MeO}$ group under the shielded zone of the nucleus, while the NOE interaction between $\mathrm{MeO}$ and $\mathrm{OH}-2^{\prime}\left(\delta_{\mathrm{H}} 5.85\right)$ clarified the same orientation of both $\mathrm{MeO}$ and $\mathrm{OH}-2^{\prime}$. Thus, the negative specific rotation of $13\left([\alpha]_{\mathrm{D}}^{25}-72\right)$ was in agreement with $2 R$ configuration.

The known compounds were determined as epicoccin $\mathrm{I}^{15}$ and epicoccin $\mathrm{A},{ }^{6}$ on the basis of comparison their spectra data with those reported in the literatures.

\subsection{Anti-HIV assay}

The isolated compounds were tested for their inhibitory effects against human immunodeficiency virus type 1 (HIV-1) replication. Anti-HIV screening was performed by the pNL4.3.Env-.Luc co-transfected 293T cells. Before bioassay, all compounds were tested for their cytotoxicity toward 293T cells, while the tested compounds showed low cytotoxicity with $\mathrm{CC}_{50}>100 \mu \mathrm{M}$. Compounds in a single dose of $20 \mu \mathrm{M}$ showing more than $50 \%$ of HIV-1 inhibition were subjected to $\mathrm{IC}_{50}$ measurement. As shown in Table 5, most of the tested compounds exerted inhibitory effects, while compound $\mathbf{5}$ showed the most inhibitory effect. A preliminary structure-activity relationship study revealed that the analogues with thiomethyl group at C-2/C-2' $(4$ and 5) showed more active than those with sulfide bridge (1-3, 6-8) in the pentacyclic thiodiketopiperazines. Comparison of the inhibitory effect between 4 and 5 revealed a double bond at C- $6^{\prime} / \mathrm{C}-7^{\prime}$ in 5 enhancing the activity. In regard to compounds 913, the analogues with thiomethyl group at C-2/C-2' (10) showed more effect than those with hydroxyl substitution (11-12), whereas the analogue with methoxy/hydroxyl substitution at C2/C-2' dramatically reduced the activity.

In addition, compound $\mathbf{1 0}$ and epicoccin A showed the reactivation on latent HIV-1 transcription with dose-dependent manner, whereas the remaining compounds exerted inactive in a dose of $100 \mu \mathrm{M}$. As shown in Fig. 5, compound 10 and epicoccin A showed the reactivation activities at $80 \mu \mathrm{M}$, which were comparable to the positive controls prostratin $(5 \mu \mathrm{M})$ and SAHA $(2.5 \mu \mathrm{M})$. Latent HIV reservoirs are the primary hurdle to eradicate human immunodeficiency virus by the highly active antiretroviral therapy (HAART), because the residual provirus
Table 5 Evaluation of anti-HIV activity of selected compounds ${ }^{a}$

\begin{tabular}{lll}
\hline Compounds & $\mathrm{IC}_{50}+\mathrm{SD}(\mu \mathrm{M})$ & $\mathrm{CC}(\mu \mathrm{M})$ \\
\hline $\mathbf{1}$ & $14.8+1.2$ & $>100$ \\
$\mathbf{2}$ & $11.5+0.8$ & $>100$ \\
$\mathbf{3}$ & $10.7+1.3$ & $>100$ \\
$\mathbf{4}$ & $8.7+0.5$ & $>100$ \\
$\mathbf{5}$ & $3.2+0.4$ & $>100$ \\
$\mathbf{6}$ & $16.6+0.5$ & $>100$ \\
$\mathbf{7}$ & $18.2+1.3$ & $>100$ \\
$\mathbf{8}$ & $13.3+0.6$ & $>100$ \\
$\mathbf{9}$ & $6.7+2.1$ & $>100$ \\
$\mathbf{1 0}$ & $4.9+1.1$ & $>100$ \\
$\mathbf{1 1}$ & $5.8+0.7$ & $>100$ \\
$\mathbf{1 2}$ & $5.9+0.9$ & $>100$ \\
$\mathbf{1 3}$ & $>20$ & $>100$ \\
EFV & 0.1 & $>100$ \\
$a$ & & \\
IC $_{50}$ (inhibitory concentration $\left.50 \%\right)$ and $\mathrm{CC}_{50}$ (cytotoxic concentration &
\end{tabular}

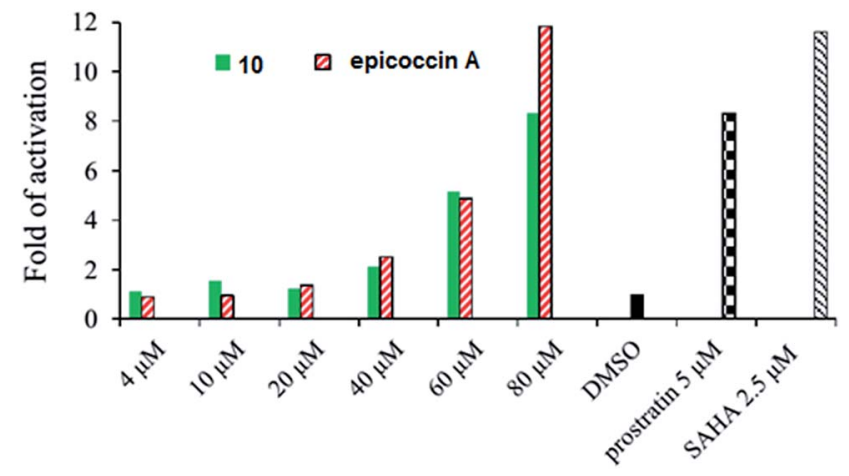

Fig. 5 Reactivating effects of 10 and epicoccin A on latent HIV-1 expression in J-Lat A2 cell. Positive control: prostratin and SAHA; negative control: DMSO. The compounds were detected by flow cytometry for the EGFP-positive cells. The activity detected in cells treated with DMSO was set to 1 and the values shown in figure were mean from two independent experiments.

harbored in cellular reservoirs quickly rebound when treatment is interrupted. ${ }^{21,22}$ One promising strategy to expunge HIV-1 infection is to reactive latent viral reservoirs in combination with HAART. ${ }^{23,24}$ Thus, finding new latency reactivating agents with noncytotoxic, clinically effective treatment of HIV infections is urgently needed.

\section{Conclusion}

Previous investigation of Eutypella fungi inhabited in terrestrial and marine habitats have uncovered diverse terpenoids ${ }^{25-28}$ and cytosporin-related compounds..$^{29,30}$ This is the first report of thiodiketopiperazine-type alkaloids to be derived from Eutypella sp., suggesting the presence of diverse biogenetic synthetic pathways in a fungal strain and the distinct synthetic pathway to be activated from a fungal strain inhabited in different location. In addition, the present work not only enriched the numbers of new analogues in thiodiketopiperazine family, also 
extended the pharmaceutical usage of thiodiketopiperazines to anti-HIV activities. The potent anti-HIV effects of the new compounds suggested that they may serve as new scaffolds for structural modification to be developed as a group of new antiHIV candidates. The reactivation on latent HIV-1 expression induced by $\mathbf{1 0}$ and epicoccin A suggested that both compounds may be applied as new latency reactivating agents, which are rarely found from natural products.

\section{Acknowledgements}

This work was supported by the grants of National High Technology and Science 973 program (2015CB755906), and NSFC (81630089, 41376127).

\section{References}

1 R. W. Timothy and M. W. Robert, Nat. Prod. Rep., 2014, 31, 1376-1404.

2 G. C. Horace, H. Karst, H. L. Robert and H. A. Byron, Agric. Biol. Chem., 1991, 55, 2037-2042.

3 Y. Zhang, S. Liu, Y. Che and X. Liu, J. Nat. Prod., 2007, 70, 1522-1525.

4 H. Guo, B. Sun, H. Gao, X. Chen, S. Liu, X. Yao, X. Liu and Y. Che, J. Nat. Prod., 2009, 72, 2115-2119.

5 L. Meng, X. Li, C. Lv, C. Huang and B. Wang, J. Nat. Prod., 2014, 77, 1921-1927.

6 Y. Sun, K. Takada, Y. Takemoto, M. Yoshida, Y. Nogi, S. Okada and S. Matsunaga, J. Nat. Prod., 2012, 75, 111-114.

7 C. Guo, H. Yeh, Y. Chiang, J. F. Sanchez, S. Chang, K. S. Bruno and C. C. C. Wang, J. Am. Chem. Soc., 2013, 135, 7205-7213.

8 H. S. Daniel, H. Andreas, H. Thorsten, A. B. Axel and H. Christian, J. Am. Chem. Soc., 2014, 136, 11674-11679.

9 R. Tan, P. R. Jensen, P. G. Williams and W. Fenical, J. Nat. Prod., 2004, 67, 1374-1382.

10 F. Kong, Y. Wang, P. Liu, T. Dong and W. Zhu, J. Nat. Prod., 2014, 77, 132-137.

11 C. Takahashi, Y. Takai, Y. Kimura, A. Numata, N. Shigematsu and H. Tanaka, Phytochemistry, 1995, 38, 155-158.

12 L. H. Meng, P. Zhang, X. M. Li and B. G. Wang, Mar. Drugs, 2015, 13, 276-287.

13 M. Saka, S. Palasarn, P. Rachtawee, S. Vimuttipong and P. Kongsaeree, Org. Lett., 2005, 7, 2257-2260.
14 H. J. Lee, J. H. Lee, B. Y. Hwang, H. S. Kim and J. J. Lee, Arch. Pharmacal Res., 2001, 25, 397-401.

15 J. Wang, G. Ding, L. Fang, J. Dai, S. Yu, Y. Wang, X. Chen, S. Ma, J. Qu, S. Xu and D. Du, J. Nat. Prod., 2010, 73, 12401249.

16 J. Wang, W. He, X. Qin, X. Wei, X. Tian, L. Liao, S. Liao, B. Yang, Z. Tu, B. Chen, F. Wang, X. Zhou and Y. Liu, RSC Adv., 2015, 5, 68736-68742.

17 J. Wang, N. Jiang, J. Ma, S. Yu, R. Tan, J. Dai, Y. Si, G. Ding, S. Ma, J. Qu, L. Fang and D. Du, Tetrahedron, 2013, 69, 11951201.

18 F. Freire, F. Calderon, J. M. Seco, A. Fernandez-Mayoralas, E. Quinoa and R. Riguera, J. Org. Chem., 2007, 72, 2297-2301.

19 N. Kawahara, K. Nozawa, S. Nakajima, M. Yamazaki and K. Kawai, Heterocycles, 1989, 29, 397-402.

20 A. Alberto, C. Silvia, N. Gianluca, V. M. Stefano and V. P. Orso, Liebigs Ann., 1996, 1875-1877.

21 B. León, G. Navarro, B. J. Dickey, G. Stepan, A. Tsai, G. S. Jones, M. E. Morales, T. Barnes, S. Ahmadyar, M. Tsiang, R. Geleziunas, T. Cihlar, N. Pagratis, Y. Tian, H. Yu and R. G. Linington, Org. Lett., 2015, 17, 262-265.

22 A. Jordan, D. Bisgrove and E. Verdin, EMBO J., 2003, 22, 1868-1877.

23 E. J. Mejia, S. T. Loveridge, G. Stepan, A. Tsai, G. S. Jones, T. Barnes, K. N. White, M. Drašković, K. Tenney, M. Tsiang, R. Geleziunas, T. Cihlar, N. Pagratis, Y. Tian, H. Yu and P. Crews, J. Nat. Prod., 2014, 77, 618-624.

24 Z. Li, J. Guo, Y. Wu and Q. Zhou, Nucleic Acids Res., 2013, 41, 277-287.

25 W. Pongcharoen, V. Rukachaisirikul, S. Phongpaichit, N. Rungjindamai and J. Sakayaroj, J. Nat. Prod., 2006, 69, 856-858.

26 L. Sun, D. Li, M. Tao, Y. Chen, F. Dan and W. Zhang, Mar. Drugs, 2012, 10, 539-550.

27 M. Isaka, S. Palasarn, W. Prathumpai and P. Laksanacharoen, Chem. Pharm. Bull., 2011, 59, 1157-1159.

28 X. Lu, J. Liu, X. Liu, Y. Gao, J. Zhang, B. Jiao and H. Zheng, J. Antibiot., 2014, 67, 171-174.

29 M. Isaka, S. Palasarn, S. Lapanun, R. Chanthaket, N. Boonyuen and S. Lumyong, J. Nat. Prod., 2009, 72, 1720-1722.

30 M. L. Ciavatta, M. P. Lopez-Gresa, M. Gavagnin, R. Nicoletti, E. Manzo, E. Mollo, Y. Guo and G. Cimino, Tetrahedron, 2008, 64, 5365-5369. 